\title{
ROCKING OF FREESTANDING OBJECTS: THEORETICAL AND EXPERIMENTAL COMPARISONS
}

\author{
Antonio Gesualdo, Antonino Iannuzzo \\ University of Naples "Federico II", Department of Structures for Engineering and Architecture, Napoli, Italy \\ e-mail: gesualdo@ununa.it; antonino.iannuzzo@unina.it
}

Vincenzo Minutolo

University of Campania "Luigi Vanvitelli", Department of Engineering, Aversa (CE), Italy

e-mail:vincenzo.minutolo@unicampania.it

Michela Monaco

University of Campania "Luigi Vanvitelli", Department of Architecture and Industrial Design, Aversa (CE), Italy

e-mail:michela.monaco@unicampania.it

The methodical study of safeguard of artistic heritage and other devices subjected to earthquake and, in general, to time-dependent forces has considerably spreaded in the last years, thus increasing researchers' interest in problems concerning motions of rigid objects simply supported on a base plane. The behaviour of piece of equipments, statues, storage tanks, or even tall buildings has been in fact studied as that of rigid bodies with relation to different base excitations. In some cases, the possibility of influencing the quality of motion can be a strong tool to reduce vulnerability, like in the cases in which rocking motion is to be avoided and sliding motion is welcome. This paper focuses the attention on this last problem. This is the same large class of both non-structural and structural elements that can lose their functionality because of earthquake motions. The results of numerical modelling of sliding and rocking motion in presence of both different excitations and mechanical parameters are presented and compared with experimental data performed by the authors. The results developed are in good agreement with the laboratory tests, and this assures the reliability of both the analytical procedure and the determination of the parameters involved.

Keywords: rocking and sliding motion, freestanding rigid body, nonlinear dynamics, experimental testing

\section{Introduction}

The limitation of excessive motion of rigid bodies under earthquake excitation is a fundamental topic in the seismic protection of art objects in museums (Erdik et al., 2010; Agbabian et al., 1991), medical devices in hospitals (Konstantinidis and Makris, 2009) and goods stored in ships. Since late XIX century, the behaviour of piece of equipments, statues, storage tanks, or even tall buildings has been studied as that of rigid bodies with relation to different base excitations (Cennamo et al., 2017). Six basic conditions (Augusti and Sinopoli, 1992) have been distinguished: rest, slide, rock, slide-rock, impact and free flight (Andreaus and Casini, 1999). Despite its familiarity and apparent simplicity, motions of rigid bodies in response to earthquake excitations pose extremely difficult problems when exact solutions are sought (Voyagaki et al., 2014). A rigid structure placed on a shaking base may enter into rocking motion that occasionally results in overturning (Guadagnuolo and Monaco, 2009). In some cases (small objects and equipments), the protection of the object can be obtained by controlling the overturning through providing an inclination to the support base in order to have a preferential direction toward a safe area (Boroshek and Iruretagoyena, 2006). 
Early studies on the rocking response of a rigid block supported on a base undergoing horizontal motion were presented by Housner (1963). To study the problem, it was necessary to first establish equations of motion of the rigid body and solve them accordingly. This was first done just by Housner following the Chilean earthquakes in 1960. Housner first considered the base motion as more irregular time functions modelled by a white noise random process. In the piecewise model by Housner, the block can only rotate around one of the base corners, and the friction coefficient is large enough to prevent any sliding. Shenton (1996) showed that the quality of motion of a rigid object simply supported on an uniformly accelerating rigid plane depended not only on the object shape and the base acceleration, but on the friction coefficient as well. According the author, friction required to initiate a rock mode increased with ground acceleration. It was shown (Monaco et al., 2014) that the range of sliding-rock was larger in the case of a harmonic excitation. The role of friction and its influence in the quality of motion was elsewhere taken into account (Sinopoli, 1997).

Since Housner had already pointed out that the rocking response of a rigid body to base motion was sensitive to the parameters defining the geometry of the body and details of base motion, Yim and Lin (1991) decided that the problem should be examined from a probabilistic point of view. Using a limited number of simulated ground motions, the authors numerically solved the equation of the problem and produced fragility curves giving the probability of the body overturn. Aslam et al. $(1978,1980)$ obtained the rocking response of a rigid body subjected to harmonic shaking as well to simulate earthquake motions both numerically and by laboratory experiments. The work of Aslam essentially confirmed the findings of Housner and Yim et al. (1980) about the difficult response prediction. In 1999, Shao and Tung followed the same approach as Yim et al. but used two types of base motions: 50 artificial earthquakes simulated in a way identical to that of Yim and 75 real earthquakes. Shao reached the same conclusion as Yim et al. but showed that real earthquakes gave less probability of overturning that simulated earthquakes did. In the first studies performed in Japan (Ishiyama, 1982), the computer simulation performed by the author showed that the horizontal velocity as well as the acceleration had to be taken into account as criteria for overturning. In engineering seismology, the rocking problem is of interest from the point of view of characterizing the ground motion level through study of overturned objects, although in many cases this practice is rather misleading and meaningless (Apostolou et al., 2007). The problem becomes simpler in the case of a massless rigid foundation as examined in Sienkiewicz (2009), where only properties of the surrounding ground are considered. The simplest mathematical models that have received notable attention in the past were the planar rocking of rigid rectangular blocks with nonzero mass under harmonic base motions (Spanos and Koh, 1984), although the influence of the ground motion properties have been analyzed since the first paper of Yim et al. above mentioned. In the harmonic problem, even in the case of a one-sine pulse (Zhang and Makris, 2001), non-linearity arises not only in the load deflection characteristic but also in dissipation of energy due to impacts (Purvance et al., 2008; Chierchiello et al., 2015).

Some studies on the response of such a system have revealed the presence of a rich variety of non-linear resonances and even the possibility of the response becoming chaotic (Bergamasco et al., 2018). Tackling analytically the equations of motion in the case of real earthquake ground motions is a non trivial task even for very simple waveforms (Konstantinidis and Makris, 2010). A range of idealized ground acceleration pulses expressed by a generalized function controlled by a single shape parameter were considered in Voyagaki et al. (2013). The problem was treated analytically by means of linearized equations of motion on the assumption of slender block geometry and rocking without sliding. The peak rocking response and overturning criteria for different waveforms were presented in terms of dimensionless closed-form expressions and graphs. The papers above mentioned (Konstantinidis and Makris, 2009, 2010) reported a series of tests in which the transient rocking response of electrical transformers subjected to horizontal 
trigonometric pulses and near source ground motion were investigated in depth. First, it was shown that the solution presented by Housner for the minimum acceleration amplitude of a half-sine pulse that was needed to overturn a rigid block was unconservative. In reality, under a half-sine pulse a block overturns during its free-vibration regime and not at the instant that the pulse expires. Within the limits of linear approximation, the correct expression that yields the minimum acceleration required to overturn a block is derived. Under realistic conditions, the rocking response of a rigid block is affected by additional factors such as the vertical component of the ground acceleration and the additional energy loss due to plastic deformations at the pivot points (Gesualdo and Monaco, 2015). Recent attempts to derive equivalence between a single rocking block and various rocking mechanisms in order to give indication for real structures has been made (Spanos et al., 2001; DeJong and Dimitrakopoulos, 2014). In general, while the rocking response under a deterministic excitation has received considerable attention (Gesualdo et al., 2014, 2018), the corresponding studies on stochastic behaviour are sparse. A significant work on stochastic analysis was carried out by Spanos and Koh (1984). They employed the statistical linearization technique to study stochastic rocking of a rigid rectangular block on Winkler's foundation due to non-stationary shaking. Koh (1986) further studied this problem through a digital simulation technique and estimated the probability of no toppling. The stochastic response of a rigid rectangular block resting on a rigid foundation and acted upon simultaneously by a horizontal and a vertical stationary white-noise excitation was considered. Recent works (Prieto and Lourenço, 2005) have tried to unify the piecewise formulation by Housner with the same hypothesis of a large friction coefficient. The traditional piecewise equations were replaced by a single ordinary differential equation, and damping effects were no longer introduced by means of a coefficient of restitution, but were understood as the presence of impulsive forces. The results are in agreement with the classical formalism, and can be set in the direct analogy with either a two-body central problem in the complex plane or an inverted pendulum through simple variable transformations. Unfortunately, these elegant formulations are unsuitable when rocking motion is to be avoided and sliding motion is welcome.

Recently, the dynamic response of a rocking block subjected to base excitation has been revisited to offer new closed-form solutions and original similarity laws that shed light on the fundamental aspects of the original model by Dimitrakopoulos and DeJong (2012). The focus is on the transient dynamics of the rocking block under finite-duration excitations. In the process, limitations of standard dimensional analysis related to the orientations of the involved physical quantities are revealed. The work shows that the nonlinear and non-smooth rocking response to pulse-type ground motion can be directly determined and need only to be scaled by the intensity and frequency of the excitation. The typology of impulses can in fact reduce or amplify the rocking response. It has been shown that in the case of multiple impulse rocking, the amplification may be significant (DeJong, 2012).

In this work, a comparison between experimental observations and theoretical models for the rocking of rigid bodies has been made. The dynamic tests elsewhere described (Monaco et al., 2014) and performed taking into account the influence of different parameters (Makris and Kampas, 2016) have been used to validate the analytical model and estimate both the restitution coefficient and the dynamic friction coefficient.

\section{Theoretical analysis}

According to the classical approach, in the response of a rigid block simply supported on a shaking plane base six basic conditions can be distinguished: rest, slide, rock, slide-rock, free flight. The analytical models revised here in the following take into account the first four classes of motion and are identified by comparing their responses with the results obtained from experimental tests on a shaking table. 


\section{1. $\quad$ Rocking model}

In this Section, the pure rocking motion is examined. Reference is made to Fig. 1, in which a rigid block with the aspect ratio $b / h$, simply supported on a moving plane is shown (Zhang and Makris, 2001).
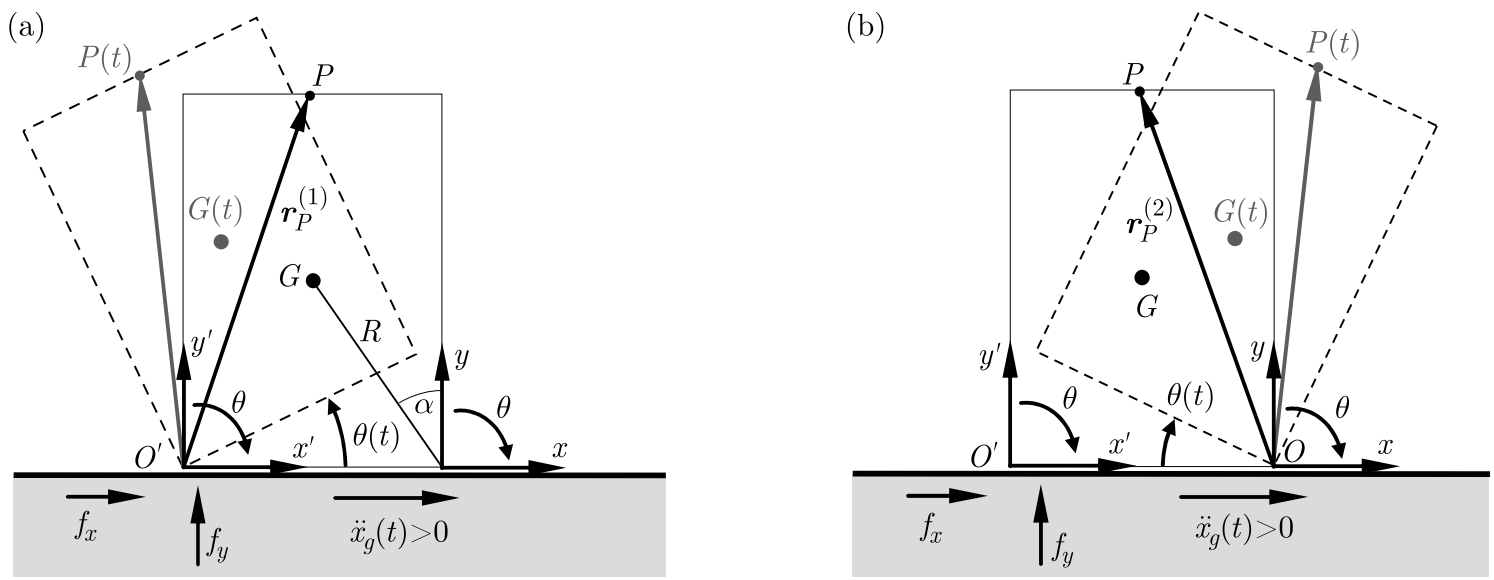

Fig. 1. Reference systems and the single block in rocking for $\theta(t)<0$ (left) and $\theta(t)>0$ (right)

The block with base $2 b$, height $2 h$ and mass $m$ can rotate alternatively around the two base corners $O$ and $O^{\prime}$, and energy is lost only during impact, when the angle of rotation $\theta(t)$ reverses. According to the classical approach of the dynamics of the inverted pendulum under impulsive excitations (Lenci and Rega, 2000), the velocity field after a perfect plastic and centered impact is related to the pre-impact field by means of the reduction coefficient $e$. In particular $e=\sqrt{r}$, being $r$ the restitution coefficient in the Housner sense. The coefficient $e$ can be assumed constant during the motion, so that the angular velocity $\dot{\theta}^{+}(t)$ of the block after the impact can be given as a function of the angular velocity $\dot{\theta}^{-}(t)$ before it (Fig. 1)

$$
\dot{\theta}^{+}(t)=e \dot{\theta}^{-}(t)
$$

In the case of the perfect plastic impact, conservation of angular momentum about the point $O^{\prime}$ just before the impact and right after the impact gives

$$
\left(I_{o}-2 m R b \sin \alpha\right) \dot{\theta}^{-}(t)=I_{o} \dot{\theta}^{+}(t)
$$

where $I_{o}$ is the moment of inertia (defined with respect to $O$ or $O^{\prime}$ ), $R$ is the distance of the center of mass $G$ from the corners $O$ or $O^{\prime}$ and $\alpha$ is the angle between $R$ and the vertical edge of the block (see Fig. 1). The combination of (2.1) and (2.2) gives for a rectangular block

$$
e=1-\frac{3}{2} \sin ^{2} \alpha
$$

Energy dissipation is involved for $e<1$. Rocking motion is present when static friction with the base plane prevents sliding. Adopting the notation by Shenton (1996), let $f_{x}$ and $f_{y}$ be the horizontal and vertical reactions at the tip $O^{\prime}$ of the block, at all times it is

$$
\left|f_{x}\right| \leqslant \mu_{s} f_{y}
$$

In other words, starting from an equilibrium configuration of the system, when the angular momentum of inertia forces is greater than that due to the gravity force, rocking motion holds 
true with condition (2.4). The rocking motion, according to d'Alembert's principle, is governed by the following relations with clockwise positive rotations

$$
\begin{array}{ll}
I_{o} \ddot{\theta}(t)+m g R \sin (-\alpha-\theta(t))=-m \ddot{x}_{g}(t) R \cos (-\alpha-\theta(t)) & \theta(t)<0 \\
I_{o} \ddot{\theta}(t)+m g R \sin (\alpha-\theta(t))=-m \ddot{x}_{g}(t) R \cos (\alpha-\theta(t)) & \theta(t)>0 \\
\dot{\theta}^{+}(t)=e \dot{\theta}^{-}(t) & \theta(t)=0
\end{array}
$$

The reference is made to Fig. 1 , where $\ddot{x}_{g}(t)$ is the horizontal base acceleration, $I_{o}$ is the inertia moment of polar inertia with respect to $O$ and the rocking motion starts when $\left|\ddot{x}_{g}(t)\right|>g b / h$. The angle $\alpha=\arctan b / h$ takes into account slenderness of the block. The resulting system of equations is composed by two ordinary nonlinear differential equations and an algebraic one relative to the impact instant. The system can assume the following simplified form

$$
\begin{array}{ll}
\frac{I_{o}}{m R} \ddot{\theta}(t)+g \operatorname{sgn}(\theta(t)) \sin [\alpha-\operatorname{sgn}(\theta(t)) \theta(t)]=-\ddot{x}_{g}(t) \cos [\alpha-\operatorname{sgn}(\theta(t)) \theta(t)] & \theta(t) \neq 0 \\
\dot{\theta}^{+}(t)=e \dot{\theta}^{-}(t) & \theta(t)=0
\end{array}
$$

with the signum function given by

$$
\operatorname{sgn}(\theta(t))= \begin{cases}+1 & \theta(t)>0 \\ -1 & \theta(t)<0\end{cases}
$$

System (2.6) has been implemented in a routine developed on purpose with the symbolic code Mathematica ${ }^{\circledR}$ (Wolfram, 2003) for a generic forcing ground acceleration. As it will be shown in the following paragraph, the experimental data have been obtained placing an accelerometer on top of the block, so that the above system should be rewritten referring to the upper face centre of the specimen. The numerical solution of differential system (2.6) may be put more conveniently in terms of the key point displacement, considering two reference systems with origins in the two rotation points $O$ and $O^{\prime}$, namely $R_{1}=\{O, x, y\}$ for $\theta(t)>0$ and $R_{2}=\left\{O^{\prime}, x^{\prime}, y^{\prime}\right\}$ for $\theta(t)<0$. The rotation matrix $R \in S O(2)$, where $S O(2)$ is the orthogonal group of matrices with $\operatorname{det}(R) \geqslant 1$, for positive clockwise rotation is

$$
R(\cdot)=\left[\begin{array}{cc}
\cos (\cdot) & \sin (\cdot) \\
-\sin (\cdot) & \cos (\cdot)
\end{array}\right]
$$

Let $\theta(t)$ be a rotation function. It is possible to evaluate the position and the acceleration of the point $P$ at the time $t$ in the two reference systems above described as

$$
\begin{array}{lll}
O P(t)=R \cdot \theta(t)\left[\begin{array}{l}
x \\
y
\end{array}\right]_{1} & \frac{\partial^{2}}{\partial t^{2}} O P(t)=\frac{d^{2}}{d t^{2}}[R \cdot \theta(t)]\left[\begin{array}{l}
x \\
y
\end{array}\right]_{1} & \theta(t)>0 \\
O^{\prime} P(t)=R \cdot \theta(t)\left[\begin{array}{l}
x \\
y
\end{array}\right]_{2} & \frac{\partial^{2}}{\partial t^{2}} O^{\prime} P(t)=\frac{d}{d t^{2}}[R \cdot \theta(t)]\left[\begin{array}{l}
x \\
y
\end{array}\right]_{2} & \theta(t)<0
\end{array}
$$

After some manipulations, the acceleration can be rewritten as follows

$$
\begin{array}{ll}
\frac{\partial^{2}}{\partial t^{2}} O P=\left[\ddot{\theta}(t) \partial R \cdot \theta(t)-\dot{\theta}^{2}(t) R \cdot \theta(t)\right]\left[\begin{array}{l}
x \\
y
\end{array}\right]_{1} & \theta(t)>0 \\
\frac{\partial^{2}}{\partial t^{2}} O^{\prime} P=\left[\ddot{\theta}(t) \partial R \cdot \theta(t)-\dot{\theta}^{2}(t) R \cdot \theta(t)\right]\left[\begin{array}{l}
x \\
y
\end{array}\right]_{2} & \theta(t)<0
\end{array}
$$

where

$$
\partial R(\cdot)=\left[\begin{array}{cc}
-\sin (\cdot) & \cos (\cdot) \\
-\cos (\cdot) & -\sin (\cdot)
\end{array}\right] \in S O(2)
$$


The horizontal component of the relative acceleration can be deduced by (2.8), and is given by

$$
\ddot{x}(t)= \begin{cases}-\left[x_{1} \cos (\theta(t))+y_{1} \sin (\theta(t))\right] \dot{\theta}^{2}(t)+\left[-x_{1} \sin (\theta(t))+y_{1} \cos (\theta(t))\right] \ddot{\theta}(t) & \theta(t)>0 \\ -\left[x_{2} \cos (\theta(t))+y_{2} \sin (\theta(t))\right] \dot{\theta}^{2}(t)+\left[-x_{2} \sin (\theta(t))+y_{2} \cos (\theta(t))\right] \ddot{\theta}(t) & \theta(t)<0\end{cases}
$$

while the absolute acceleration is

$$
\ddot{x}_{a}(t)=\ddot{x}_{g}(t)+\ddot{x}(t)
$$

\section{2. $\quad$ Sliding model}

The configuration of the block in the case of sliding motion can be characterized by translation of a generic point of the block with respect to the base. The friction force is a function of vertical forces applied to the block and is opposite to the motion.

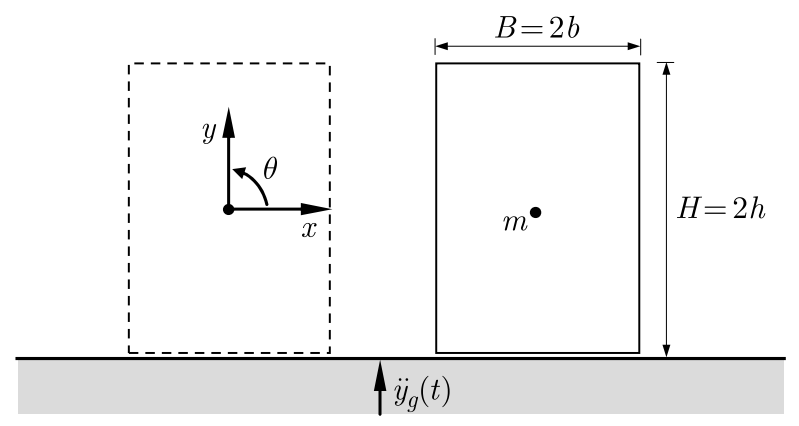

Fig. 2. The rigid block. Sliding motion

Starting from an equilibrium configuration, sliding motion begins when the maximum horizontal force due to the static friction coefficient is attained

$$
m\left|\ddot{x}_{g}(t)\right|>m \mu_{s}\left[\ddot{y}_{g}(t)+g\right]
$$

The differential equation governing the sliding motion problem is

$$
m\left[\ddot{x}_{g}(t)+\ddot{x}(t)\right]=-\operatorname{sgn}(\dot{x}(t)) \mu_{k} m\left[\ddot{y}_{g}(t)+g\right]
$$

with

$$
\operatorname{sgn}(\dot{x}(t))= \begin{cases}+1 & \dot{x}(t)>0 \\ -1 & \dot{x}(t)<0\end{cases}
$$

The numerical procedure has been developed in the general case of nonzero vertical acceleration of the base whose equations are deducted by (2.11) and (2.12) with $\ddot{y}_{g}(t)=0$

$$
m\left|\ddot{x}_{g}(t)\right|>m g \mu_{s} \quad m\left[\ddot{x}_{g}(t)+\ddot{x}(t)\right]=-\operatorname{sgn}(\dot{x}(t)) m g \mu_{k}
$$

Starting from the instant in which the contact force due to static friction is exceeded (2.10), differential equation of sliding (2.11) is integrated until the relative velocity $\dot{x}(t)$ is nonzero. When the velocity becomes null, the block is in relative equilibrium with the base (rest) until the external force attains a value able to reactivate the sliding motion. 


\section{Experimental tests}

The behaviour of prismatic specimens subjected to harmonic shaking is investigated. The results of the above analytical model are compared with experimental tests developed by means of an unidirectional shaking table on prismatic specimens (Monaco et al., 2014). The specimens, prisms with two different aspect ratios, were made of two different materials: cellular concrete and basalt (Fig. 3).
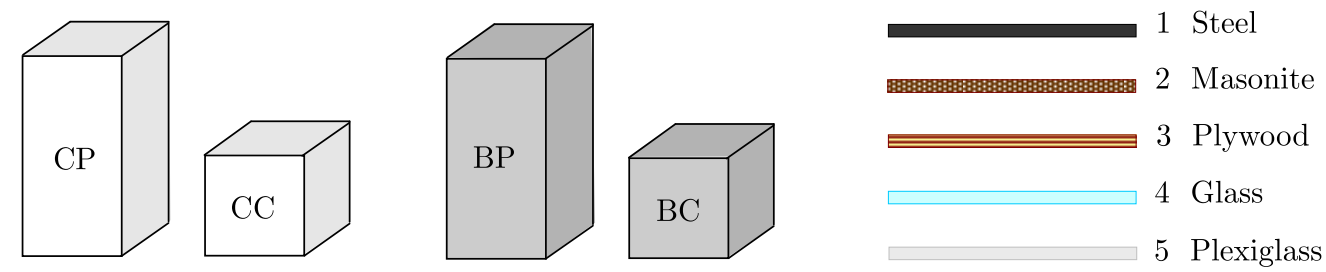

Fig. 3. Specimens respectively made of cellular concrete (C, left) and basalt (B, center), with cubic (C) and prismatic $(\mathrm{P})$ shape and five types of support (right)

On the shaking table plane, five different material supports were arranged, in order to analyze the influence of three different parameters: aspect ratio, friction coefficient and base motion frequency. The shaking table was in fact able to perform a horizontal harmonic excitation with a variable frequency up to $10 \mathrm{~Hz}$ and a constant span of $1.5 \mathrm{~mm}$.

(a)

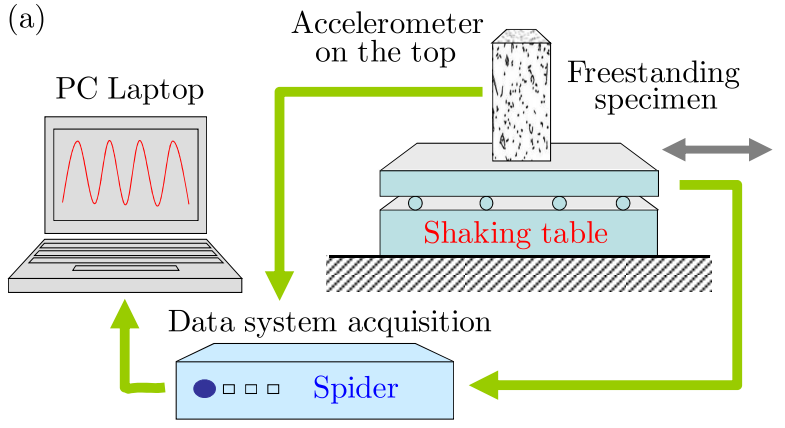

(b)

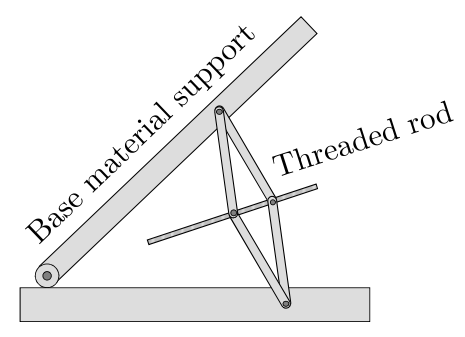

Fig. 4. Shaking table and the acquisition system (left) and the test device for determination of the friction coefficient (right)

As a result, combinations of ten different friction coefficients determined with an on purpose designed test device, and two different aspect ratios $(b / h=1$ for cubic specimens and $b / h=0.5$ for prismatic ones), were taken into account.

The friction coefficient has been determined by means of a test device made of a steel structure in which the upper plane could be inclined by means of a worm gear that could be manually controlled. The specimen, equipped with an accelerometer, was placed on the apparatus to which the support material had been previously fixed. The inclination of the support plane was increased slowly until the beginning of specimen sliding (Fig. 4). The values of the friction coefficients so determined were considered as static friction coefficients. They are reported in Table 1 for basalt and cellular concrete specimens, respectively. Shenton (1996) developed simple criteria to evaluate the initial motion of freestanding symmetric objects on a moving base with the acceleration described by a piecewise continuous function ranging from zero (base at rest) to the value $A_{g}$ (peak value). The author divided the space $A_{g}-\varphi$ into four regions corresponding to the four possible motions: rest, slide, rocking, slide-rock 


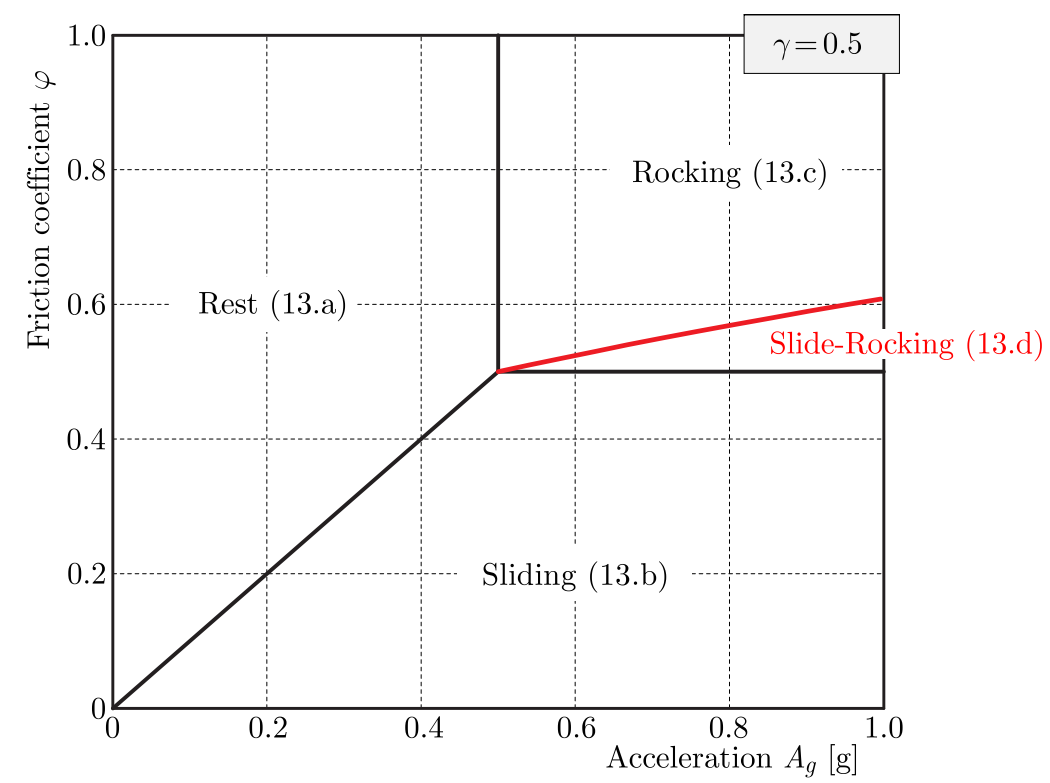

Fig. 5. Four Shenton's regions with the proposed enlargement in the case of the sinusoidal base excitation

$$
\left.\left.\begin{array}{l}
\left.\begin{array}{l}
A_{g} \leqslant \varphi \\
A_{g} \leqslant \gamma
\end{array}\right\} \text { rest } \\
\left.\begin{array}{l}
A_{g}>\varphi \\
\varphi<\gamma
\end{array}\right\} \text { slide } \\
A_{g} \leqslant \frac{\left(1+4 \gamma^{2}\right) \varphi-3 \gamma}{\left(4+\gamma^{2}\right)-3 \gamma \varphi}
\end{array}\right\} \begin{array}{l}
\text { rocking } \\
A_{g}>\frac{\left(1+4 \gamma^{2}\right) \varphi-3 \gamma}{\left(4+\gamma^{2}\right)-3 \gamma \varphi}
\end{array}\right\} \text { slide/rock }
$$

where $\gamma$ is the aspect ratio, $\gamma=b / h$ and $A_{g}$ is the peak ground acceleration. The criteria, expressed by relations (3.1) are represented in Fig. 5. A tentative description of the variation of the Shenton regions for the sinusoidal base acceleration was proposed in (Monaco et al., 2014). It was shown that in the last case, expansion of the slide-rocking area with respect to that fixed by Shenton was observed (Fig. 5). The expanded slide-rocking area proposed is the following

$$
\left.\begin{array}{l}
A_{g}>\gamma \\
A_{g}>\frac{\left(1+4 \gamma^{2}\right) \varphi-3 \gamma}{\left(4+\gamma^{2}\right)-3 \gamma \varphi}
\end{array}\right\} \quad \text { slide/rock }
$$

The results of the experimental tests confirm the expansion of the slide-rocking area for the harmonic base acceleration: Figure 6 represents two different Shenton planes corresponding to the two aspect ratios examined.

It can be noted that, according to the Shenton scheme, for the unitary aspect ratio, the rocking area should be absent, but the experiments have shown the presence of all motion for cubic specimens (rest, sliding and rocking). Similarly, in the case of prismatic specimens, the experiments have shown the presence of all motion (rest, sliding and slide-rocking). The enlargement of the slide-rocking area is reported in Fig. 6 right, where the red line is the boundary of the enlarged area represented by relation (3.2). The experimental results were obtained placing the specimen on the shaking table to which the support material had been previously fixed. An accelerometer was placed on the top of the specimen to evaluate the accelerations registered together with the shaking table data by means of a computer acquisition data system (Fig. 4). The laboratory time histories are discussed and compared with the analytical ones in the following Section. 

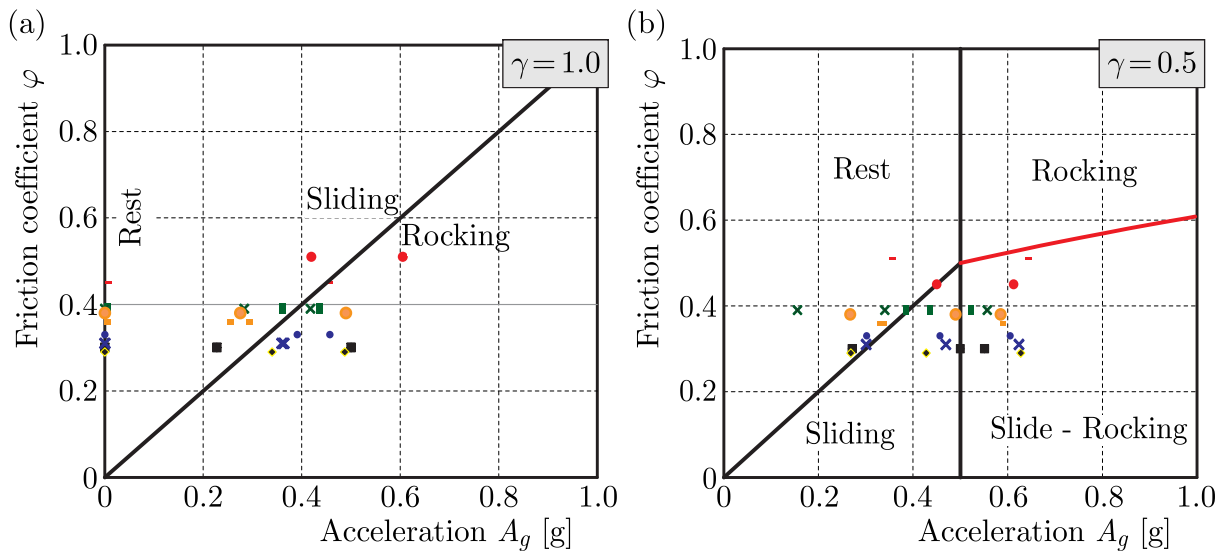
$-A_{g}=\frac{\left(1+4 \gamma^{2}\right) \varphi-3 \gamma}{\left(4+\gamma^{2}\right)-3 \gamma \varphi}$
- Concrete - Masonite
- Basalt - Masonite
- Concrete - Steel
$\times$ Basalt - Steel
- Concrete - Plywood
- Basalt - Plywood
Concrete - Plexiglass
$\times$ Basalt - Plexiglass
Concrete - Glass
Basalt - Glass

Fig. 6. Friction coefficient vs. peak specimen acceleration for cubic blocks $(\gamma=1$, left) and prismatic blocks $(\gamma=0.5$, right $)$

\section{Numerical comparisons}

The laboratory test conditions are similar to the real cases of freestanding art objects in museums or laboratory equipments in hospitals, where friction coefficients are of various types due to different quality of support planes (pedestals, shelves, floor). In order to provide a ductile analytical tool for the safeguard of such objects, the numerical procedure previously described in Section 2 has been on purpose implemented in the Mathematica ${ }^{\circledR}$ (2003) routine. Theoretical curves have been obtained by taking into account material parameters evaluated in the experimental tests and reported in Table 1.

Table 1. Static and dynamic friction coefficients, restitution coefficients and frequencies of the examined time histories

\begin{tabular}{|c|c|c|c|c|c|c|}
\hline $\begin{array}{c}\text { Motion } \\
\text { type }\end{array}$ & $\begin{array}{l}\text { Specimen } \\
\text { type }\end{array}$ & $\begin{array}{l}\text { Support } \\
\text { material }\end{array}$ & \begin{tabular}{|c} 
Static friction \\
coefficient
\end{tabular} & $\begin{array}{c}\text { Dynamic friction } \\
\text { coefficient }\end{array}$ & $\begin{array}{c}\text { Restitution } \\
\text { coefficient }\end{array}$ & $\begin{array}{r}\text { Frequency } \\
\text { examined }\end{array}$ \\
\hline & & 1 & 0.31 & - & 0.925 & 9.2 \\
\hline \multirow{3}{*}{ Rocking } & \multirow{3}{*}{$\mathrm{BP}$} & 2 & 0.29 & - & 0.925 & 9.2 \\
\hline & & 4 & 0.38 & - & 0.955 & 14.3 \\
\hline & & 5 & 0.39 & - & 0.955 & 14.2 \\
\hline \multirow{4}{*}{ Sliding } & \multirow{4}{*}{$\mathrm{CP}$} & 1 & 0.30 & 0.26 & - & 6.3 \\
\hline & & 2 & 0.29 & 0.25 & - & 6.7 \\
\hline & & 4 & 0.33 & 0.29 & - & 7.2 \\
\hline & & 5 & 0.30 & 0.26 & - & 7.2 \\
\hline \multirow{4}{*}{ Sliding } & \multirow{4}{*}{$\mathrm{BP}$} & 1 & 0.31 & 0.27 & - & 7.0 \\
\hline & & 2 & 0.29 & 0.25 & - & 7.5 \\
\hline & & 4 & 0.38 & 0.33 & - & 8.3 \\
\hline & & 5 & 0.39 & 0.34 & - & 7.5 \\
\hline \multirow{4}{*}{ Sliding } & \multirow{4}{*}{$\mathrm{CC}$} & 1 & 0.33 & 0.29 & - & 9.3 \\
\hline & & 3 & 0.45 & 0.40 & - & 8.8 \\
\hline & & 4 & 0.33 & 0.29 & - & 11.0 \\
\hline & & 5 & 0.30 & 0.26 & - & 8.3 \\
\hline \multirow{4}{*}{ Sliding } & \multirow{4}{*}{$\mathrm{BC}$} & 1 & 0.31 & 0.27 & - & 10.0 \\
\hline & & 2 & 0.29 & 0.26 & - & 8.0 \\
\hline & & 3 & 0.51 & 0.45 & - & 9.0 \\
\hline & & 4 & 0.36 & 0.32 & - & 8.7 \\
\hline
\end{tabular}


Both restitution and dynamic friction coefficients used in the analytical rocking model are reported. They are satisfactorily in good agreement with the experimental data. In particular, the value of the dynamic friction coefficient used is $88 \%$ of the static friction coefficient measured in the laboratory tests, according to the literature, usual values (Bryant, 2016). In Figs. 7-11, time histories obtained in the numerical and experimental campaign are reported: the red line gives the analytical results while the grey one the experimental data. It must be noted that part of the mechanical parameters has been estimated, nevertheless, the numerical model is capable of representing the quality of motion with great accuracy.
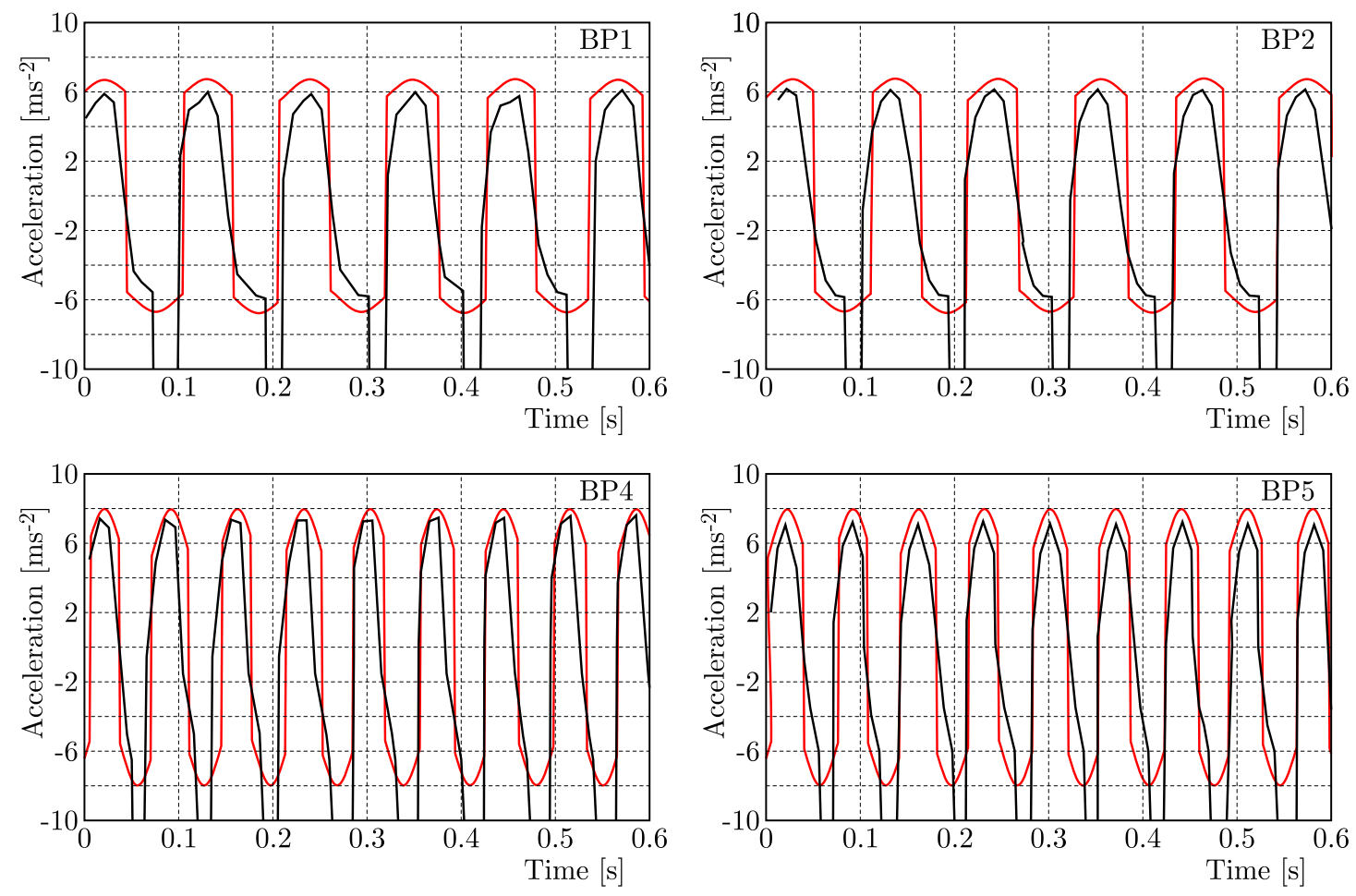

Fig. 7. Rocking motion: analytical (red) and experimental (grey) acceleration time histories

The numerical diagrams obtained are in good agreement with the experimental ones, see for example diagrams BP2 and BP4 in Fig. 8 (basalt prisms) and diagrams $\mathrm{BC} 2$ and $\mathrm{BC} 4$ (basalt cubes) in Fig. 9 or diagrams CP4 and CP5 in Fig. 10 (concrete prisms) and diagrams CC4 and CC5 (concrete cubes) in Fig. 11. The presence of the accelerometer on the top of the specimen and the acquisition wire gives in general small variation in a single cycle. The analytical curves present small discontinuities due to the structure of the governing equations, but in general the dynamic behaviour is well identified. This confirms that the only estimated parameter, i.e. the dynamic friction coefficient reported in Table 1, has been correctly evaluated. In fact, a reduction of $12 \%$ with respect to the static friction coefficient has been experimentally determined. The experimental determination of the dynamic friction coefficient and realization of dynamic tests are somewhat difficult, so that the theoretical model is the starting point for an optimization in the case of safeguard of freestanding objects. It must be considered that, nowadays, it is possible to make surfaces in which the friction coefficient can be previously determined, so that a vulnerable object can be endowed with a specific base surface in order to obtain, in contact with the support plane, a fixed friction coefficient. The motion can in this case be controlled in the case of strong earthquakes and motions that can cause more damage, i.e. rocking ones can be avoided. As expected from the general mechanic statements and verified experimentally, the static friction coefficient is independent of the shape of the specimen and depends only on the contact surfaces. The analysis of experimental diagrams has been taken into account in the 

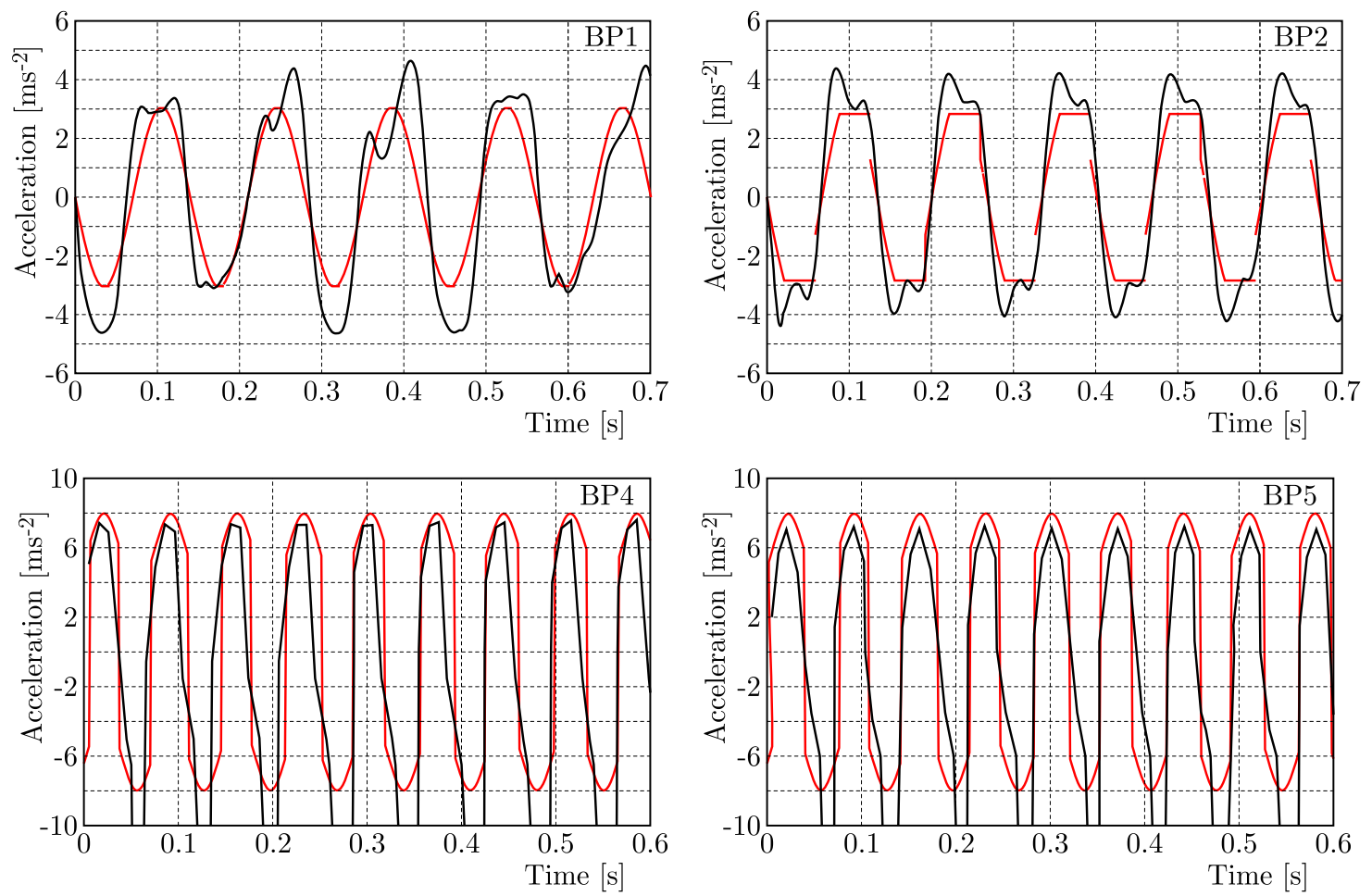

Fig. 8. Sliding motion: analytical (red) and experimental (grey) acceleration time histories
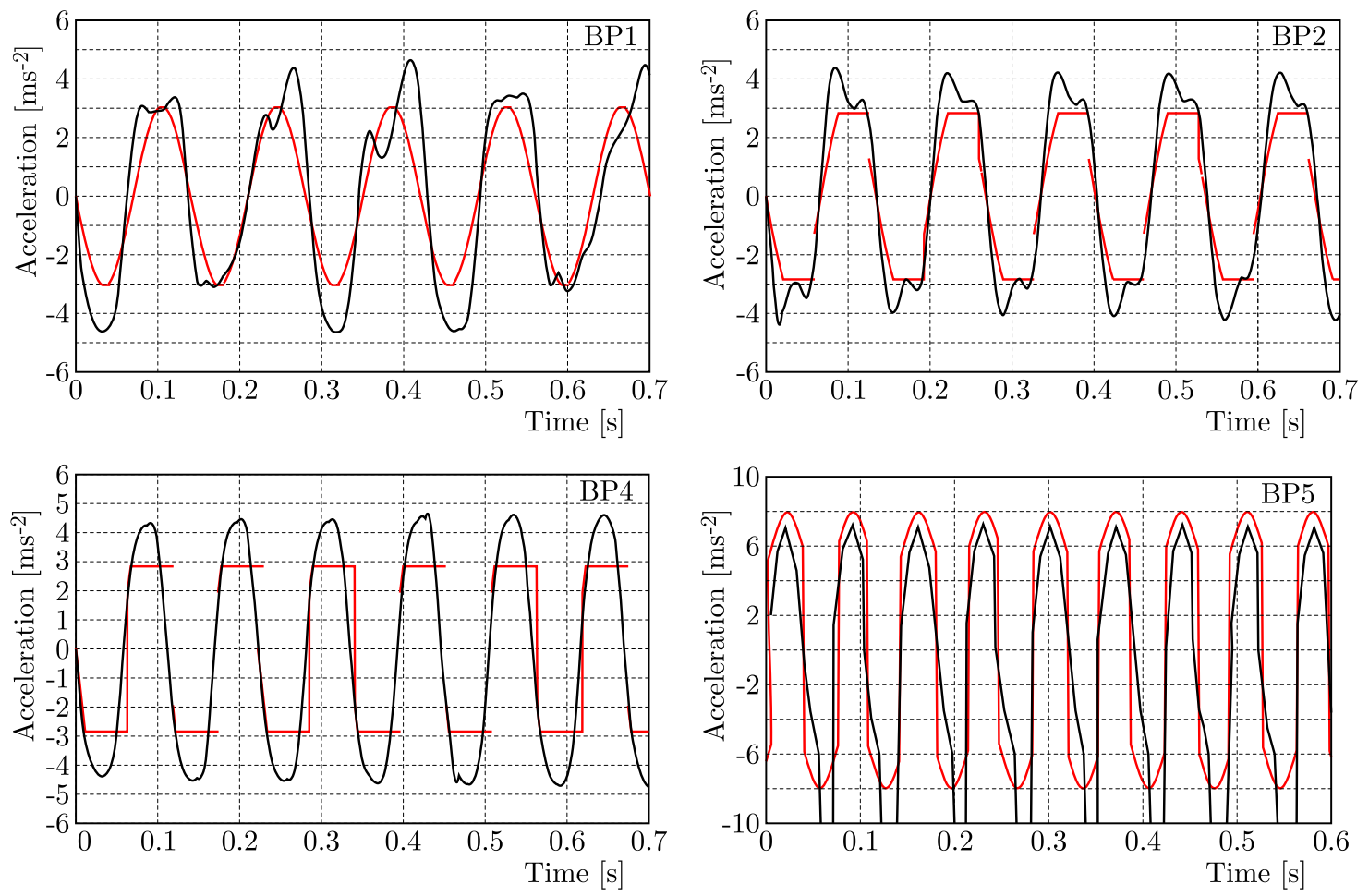

Fig. 9. Sliding motion: analytical (red) and experimental (grey) acceleration time histories

estimation of the dynamic friction coefficient, so that an unique parameter for the aspect ratio has been considered in the numerical procedure. It must be noted that the use of two different values for dynamic and static friction coefficients is the reason of jumps in the analytical curves. 

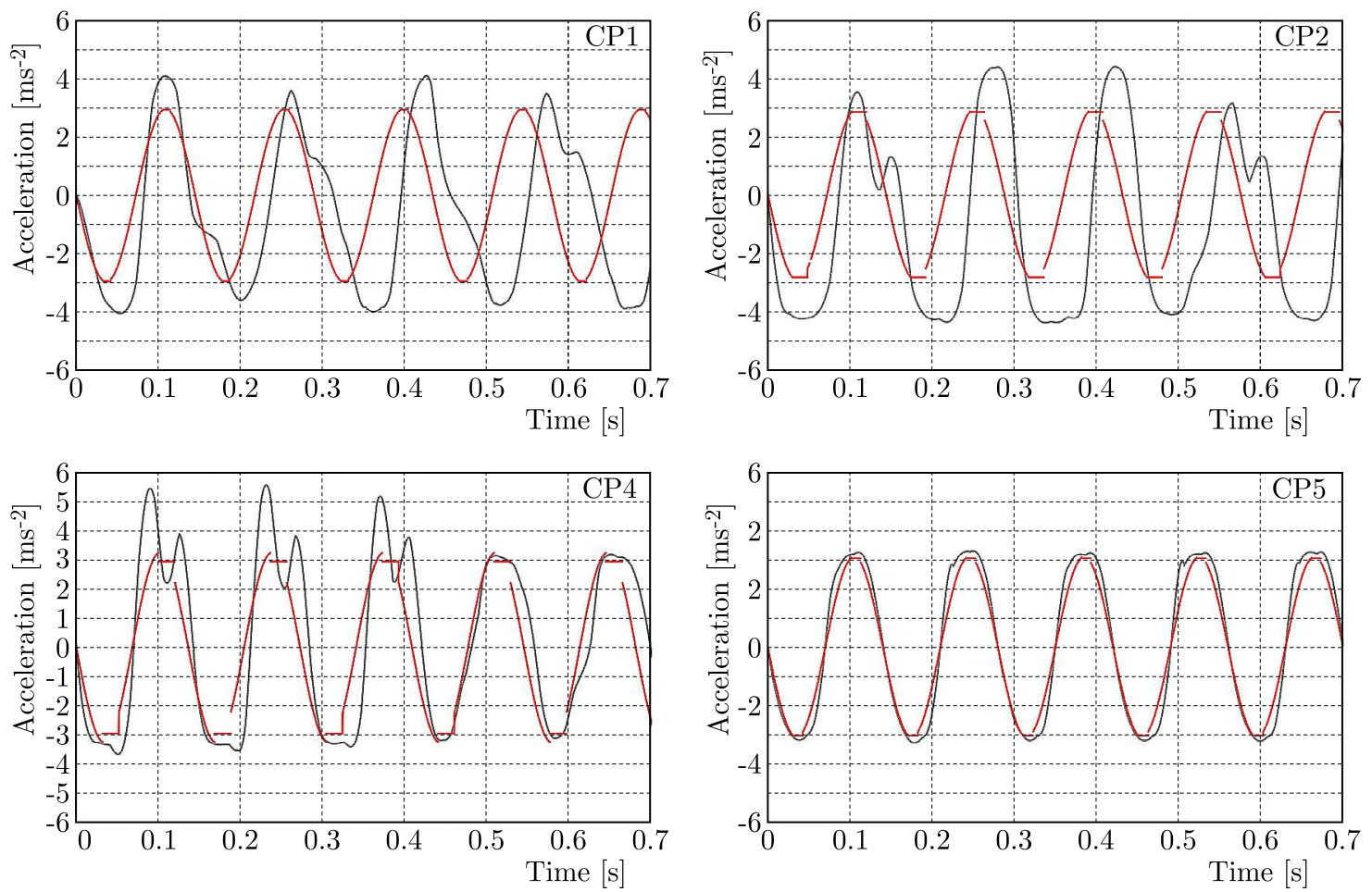

Fig. 10. Sliding motion: analytical (red) and experimental (grey) acceleration time histories
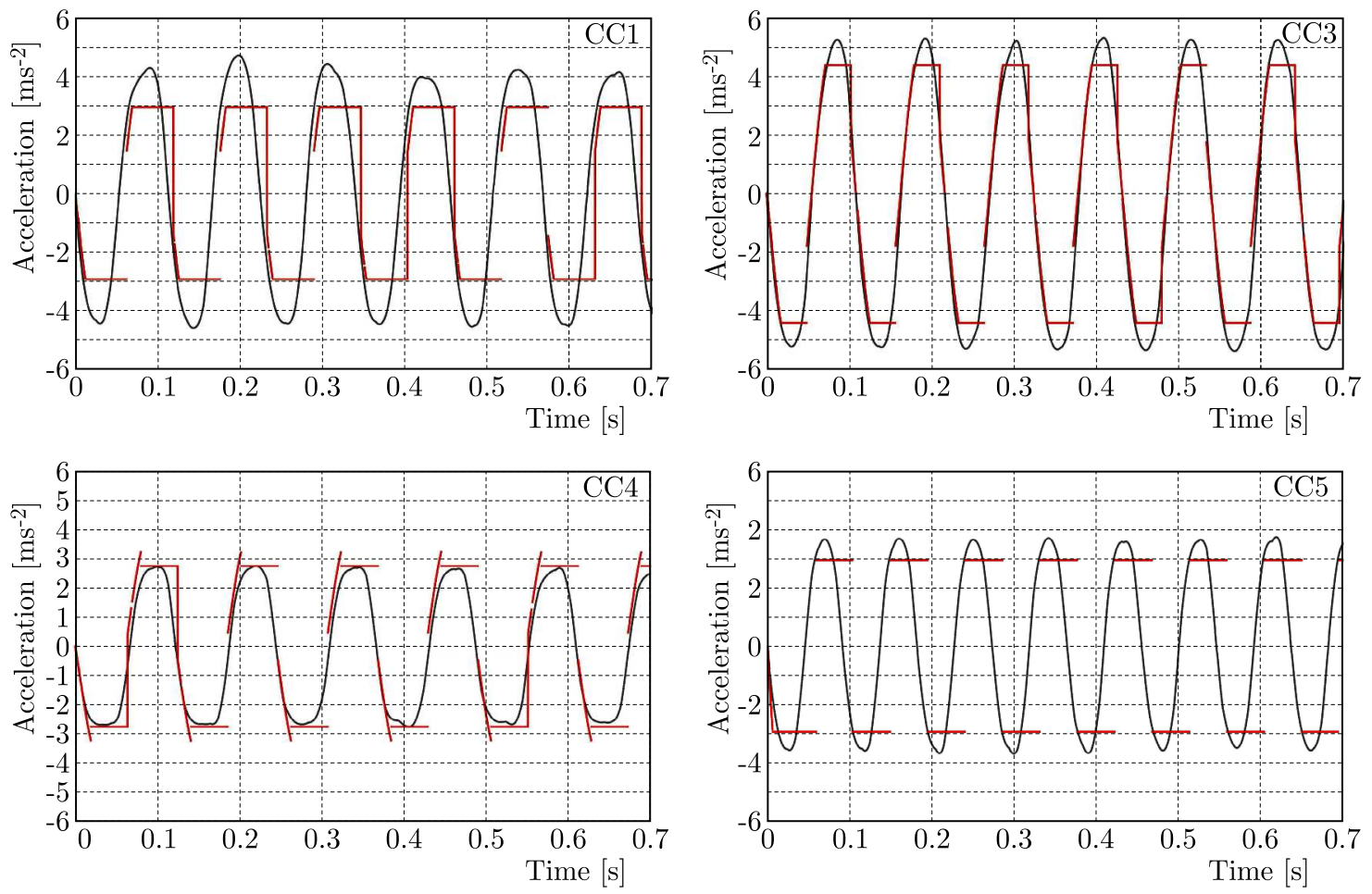

Fig. 11. Sliding motion: analytical (red) and experimental (grey) acceleration time histories

\section{Conclusions}

The problem of a freestanding rigid object on a moving base has been investigated. The results of numerical modelling of both sliding and rocking motion have been presented and compared with experimental data obtained by the authors on rigid blocks simply supported on a rigid ground 
with different friction coefficients between the object and the supporting plane and different aspect ratios of the rigid object. The numerical procedure involves all possible types of motion and takes into account static and dynamic friction coefficients. While the first one has been experimentally derived, the second one has been estimated as a percent of the first, both the values being independent of the shape of the rigid object tested. It has been shown that in the range of frequencies of the laboratory tests performed, a reduction by $12 \%$ of the static friction coefficient gives a reliable value of the dynamic one. Laboratory tests and numerical results are in good agreement, which confirms that the theoretical model is the starting point for an optimization in the case of safeguard of freestanding objects. It must be emphasized that, nowadays, it is possible to design surfaces in which the friction coefficient can be previously determined, so that a vulnerable object can be endowed with a specific base surface in order to obtain, in contact with the support plane, a fixed friction coefficient. The motion can in this case be controlled in the case of strong earthquakes and motions that can cause more damage, i.e. rocking ones, can be avoided. Since the experimental tests are often difficult as well as expensive, especially in the case of art objects and ancient artifacts, the development of a reliable analytical procedure and simple determination of mechanical parameters involved in the correct interpretation of the problem is very useful.

\section{References}

1. Agbabian M.S., Ginell W.S., Masri F.S., Nigbor R.L., 1991, Evaluation of earthquake damage mitigation methods for museum objects, Studies in Conservation, 36, 1, 111-120, DOI: 10.1179/sic.1991.36.2.111

2. Andreaus U., Casini P., 1999, On the rocking-uplifting motion of a rigid block in free and forced motion: influence of sliding and bouncing, Acta Mechanica, 138, 3-4, 219-41, DOI: 10.1007/BF01291846

3. Apostolou M., Gazetas G., Garini E., 2007, Seismic response of slender rigid structures with foundation uplifting, Soil Dynamics and Earthquake Engineering, 27, 7, 642-654, DOI: 10.1016/j.soildyn.2006.12.002

4. Aslam M., Godden W., Scalise D., 1978, Rocking and Overturning Response of Rigid Bodies to Earthquake Motions, Lawrence Berkeley National Laboratory, University of California, Berkeley, USA

5. Aslam M., Scalise D.T., Godden W.G., 1980, Earthquake rocking response of rigid bodies, Journal of the Structural Division ASCE, 106, 2, 377-392

6. Augusti G., Sinopoli A., 1992, Modelling the dynamic of large block structures, Meccanica, 27, 3, 195-211, DOI: 10.1007/BF00430045

7. Bergamasco I., Gesualdo A., Iannuzzo A., Monaco M., 2018, An integrated approach to the conservation of the roofing structures in the Pompeian Domus, Journal of Cultural Heritage, 31, 141-151, DOI: 10.1016/j.culher.2017.12.006

8. Boroshek R., Iruretagoyena A., 2006, Controlled overturning of unanchored rigid bodies, Earthquake Engineering and Structural Dynamics, 35, 6, 695-711, DOI: 10.1002/eqe.554

9. Bryant M.D., 2016, On constitutive relations for friction from thermodynamics and dynamics, ASME Journal of Tribology, 138, 4, 1215-1223, DOI: 10.1115/1.4032821

10. Cennamo C., Gesualdo A., Monaco M., 2017, Shear plastic constitutive behaviour for near-fault ground motion, Journal of Engineering Mechanics ASCE, 143, 9, 04017086, DOI: 10.1061/(ASCE)EM.1943-7889.0001300

11. Chierchiello G., Gesualdo A., Iannuzzo A., Monaco M., Savino M.T., 2015, Structural modeling and conservation of single columns in archaeological areas, Proceedings of XIV International Forum "Le vie dei mercanti", Aversa- Capri, Italy, La Scuola di Pitagora Editrice, Napoli, Italy, 2012-2020 
12. DeJong M.J., 2012, Amplification of rocking due to horizontal ground motion, Earthquake Spectra, 28, 4, 1405-1421, DOI: 10.1193/1.4000085

13. DeJong M.J., Dimitrakopoulos E.G., 2014, Dynamically equivalent rocking structures, Earthquake Engineering and Structural Dynamics, 43, 10, 1543-1564, DOI: 10.1002/eqe.2410

14. Dimitrakopoulos E.G., DeJong M.J., 2012, Revisiting the rocking block: Closed form solutions and similarity laws, Proceedings of the Royal Society A, 468, 2144, 2294-2318, DOI: 10.1098/rspa.2012.0026

15. Erdik M., Durukal E., Ertürk N., Sungay B., 2010, Earthquake risk mitigation in Istanbul museums, Natural Hazards, 53, 1, 97-108, DOI: 10.1007/s11069-009-9411-2

16. Gesualdo A., Innnuzzo A., Monaco M., Penta F., 2018, Rocking of a rigid block freestanding on a flat pedestal, Journal of Zhejiang University Science A, 19, 5, 331-345, DOI: $10.1631 /$ jzus.A1700061

17. Gesualdo A., Iannuzzo A., Monaco M., Savino M.T., 2014, Dynamic analysis of freestanding rigid blocks, [In:] Civil-Comp Proceedings of the Twelfth International Conference on Computational Structures Technology, B.H.V. Topping and P. Iványi (Eds.), 106, Civil Comp Press, Kippen, Stirlingshire, U.K., DOI: 10.4203/ccp.106.144

18. Gesualdo A., Monaco M., 2015, Constitutive behaviour of quasi-brittle materials with anisotropic friction, Latin American Journal of Solids and Structures, 12, 4, 695-710, DOI: 10.1590/167978251345

19. Guadagnuolo M., Monaco M., 2009, Out of plane behaviour of unreinforced masonry walls, [In:] Protection of Historical Buildings, 2, 1177-1180, London, New York: CRC Press, Taylor \& Francis Group

20. Housner W.G., 1963, The behaviour of inverted pendulum structures during earthquake, Bulletin of the Seismological Society of America, 53, 2, 403-417

21. Ishiyama Y., 1982, Motions of rigid bodies and criteria for overtuning by earthquake excitations, Earthquake Engineering and Structural Dynamics, 10, 5, 635-650, DOI: 10.1007/s11069-014-1076-9

22. Кон A.S., 1986, Rocking of rigid bodies on randomly shaking foundations, Nuclear Engineering and Design, 97, 2, 269-276, DOI: 10.1016/0029-5493(86)90114-7

23. Konstantinidis D., Makris N., 2009, Experimental and analytical studies on the response of freestanding laboratory equipment to earthquake shaking, Earthquake Engineering and Structural Dynamics, 38, 6, 827-848, DOI: 10.1002/eqe.871

24. Konstantinidis D., Makris N., 2010, Experimental and analytical studies on the response of a $1 / 4$ scale model of freestanding laboratory equipment subjected to strong earthquake shaking, Bulletin of Earthquake Engineering, 8, 6, 1457-1477, DOI: 10.1007/s10518-010-9192-8

25. Lenci S., Rega G., 2000, Periodic solutions and bifurcations in an impact inverted pendulum under impulsive excitation, Chaos, Solitons and Fractals, 11, 5, 2453-2472, DOI: 10.1016/S09600779(00)00030-8

26. MAKRIS N., Kampas G., 2016, Size versus slenderness: two competing parameters in the seismic stability of free-standing rocking columns, Bulletin of the Seismological Society of America, 106, 1, 104-122, DOI: 10.1785/0120150138

27. Monaco M., Guadagnuolo M., Gesualdo A., 2014, The role of friction in the seismic risk mitigation of freestanding art objects, Natural Hazards, 73, 2, 389-402, DOI: 10.1007/s11069-0141076-9

28. Prieto F., Lourenço P.B., 2005, On the rocking behaviour of rigid objects, Meccanica, 40, 2, 121-133, DOI: 10. 1007/s11012-004-5875-Z

29. Purvance M.D., Abdolrasool A., Brune J.N., 2008, Freestanding block overturning fragilities: numerical simulation and experimental validation, Earthquake Engineering and Structural Dynamics, 37, 5, 791-808, DOI: 10.1002/eqe.789 
30. Shao Y., Tung C.C., 1999, Seismic response of unanchored bodies, Earthquake Spectra, 15, 3, 523-536, DOI: 10.1193/1.1586056

31. Shenton H.W., 1996, Criteria for initiation of slide, rock, and slide-rock rigid-body modes, Journal of Engineering Mechanics ASCE, 122, 7, 690-693, DOI: 10.1061/(ASCE)07339399(1996)122:7(690)

32. Sienkiewicz Z., 2009, Local modelling of backfill effects for rigid axisymmetric foundations under dynamic excitation, Journal of Theoretical and Applied Mechanics, 47, 4, 923-942

33. Sinopoli A., 1997, Unilaterality and dry friction: a geometric formulation for two-dimensional rigid body dynamics, Nonlinear Dynamics, 12, 4, 343-366, DOI: 10.1023/A:1008289716620

34. Spanos P., Кон A.S., 1984, Rocking of rigid blocks due to harmonic shaking, Journal of Engineering Mechanics ASCE, 110, 11, 1627-1642, DOI: 10.1061/(ASCE)0733-9399(1984)110:11(1627)

35. Spanos P.D., Roussis P.C., Politis N.P.A., 2001, Dynamic analysis of stacked rigid blocks, Soil Dynamics and Earthquake Engineering, 21, 7, 559-578, DOI: 10.1016/S0267-7261(01)00038-0

36. Voyagaki E., Psycharis I., Mylonakis G., 2013, Rocking response and overturning criteria for free standing rigid blocks to single-lobe pulses, Soil Dynamics and Earthquake Engineering, 46, 85-95, DOI: 10.1016/j.soildyn.2012.11.010

37. Voyagaki E., Psycharis I., Mylonakis G., 2014, Complex response of a rocking block to a full-cycle pulse, Journal of Engineering Mechanics ASCE, 140, 6, 04014024, DOI: 10.1061/(ASCE)EM.1943-7889.0000712, 04014024

38. Wolfram S., 2003, The Mathematica Book, Wolfram Media, USA

39. Yim S.C.S., Chopra A., Penzien J., 1980, Rocking response of rigid blocks to earthquakes, Earthquake Engineering and Structural Dynamics, 8, 6, 565-580, DOI: 10.1002/eqe.4290080606

40. Yim S.C.S., Lin H., 1991, Nonlinear impact and chaotic response of slender rocking objects, Journal of Engineering Mechanics ASCE, 117, 9, 2079-2100, DOI: 10.1061/(ASCE)07339399(1991)117:9(2079)

41. Zhang J., Makris N., 2001, Rocking response of free-standing blocks under cycloidal pulses, Journal of Engineering Mechanics ASCE, 127, 5, 473-483, DOI: 10.1061/(ASCE)07339399(2001)127:5(473) 